\title{
Albendazole Microparticles Prepared by Spray Drying Technique: Improvement of Drug Dissolution
}

\author{
Mohamed Abbas Ibrahim ${ }^{1,4}$, Gamal A Shazly ${ }^{2,4 *}$ and Mahmoud El-Badry,4 \\ ${ }^{1}$ Department of Pharmaceutics and Industrial Pharmacy, Faculty of Pharmacy, Al-Azhar University, ${ }^{2}$ Department of Industrial \\ Pharmacy, Faculty of Pharmacy, ${ }^{3}$ Department of Pharmaceutics, Faculty of Pharmacy, Assiut University, Assiut, Egypt, \\ ${ }^{4}$ Department of Pharmaceutics, College of Pharmacy, King Saud University, PO Box 2457, Riyadh 11451, Saudi Arabia
}

*For correspondence: Email: gamalmym@gmail.com; Tel: +966590447099

Received: 11 October 2013

Revised accepted: 23 June 2014

\begin{abstract}
Purpose: To enhance the dissolution of albendazole (ABZ) using spray-drying technique. Method: $A B Z$ binary mixtures with Kollicoat IR@ $(K L)$ and polyvinyl pyrrolidone $(P V P)$ in various drug to polymer ratios (1: 1, 1: 2 and 1; 4) were prepared by spray-drying. The spray-dried particles were characterized for particle shape, and dissolution rate as well as by differential scanning calorimetry(DSC) and Fourier transform infrared (FTIR).

Results: Scanning electron micrographs showed a homogeneous distribution of ABZ in the polymer matrix for ABZ-PVP spray-dried system in ratios of 1:2 and 1: 4, while it was observed only upon using a ratio of ABZ: KL 1: 4 in case of ABZ-KL systems. FT-IR spectra of both physical mixtures and spraydried mixtures did not show any change for all ABZ-polymer systems, thus indicating the compatibility of the carriers with $A B Z$. ABZ exhibited a noticeable enhanced dissolution rate from its spray-dried coacervate with PVP and this was independent of the drug/polymer ratio. Drug release was 78, 81 and $81 \%$ from the spray-dried ABZ-PVP systems of drug: polymer ratio of 1:1, 1:2 and 1: 4, respectively, within $5 \mathrm{~min}$. Drug showed complete dissolution within $15 \mathrm{~min}$. On the other hand, enhancement of dissolution rate varied with $A B Z$ : $K I$ ratio.

Conclusion: Enhancement of $A B Z$ dissolution for both types of spray-dried particles is due to the reduction in drug particle sizes, wetting of the dissolution medium by the hydrophilic carriers and the amorphosization of the drug crystals by the carriers.
\end{abstract}

Keywords: Albendazole, Spray-drying, Kollicoat, Polyvinyl pyrrolidone, Dissolution, Amorphous

Tropical Journal of Pharmaceutical Research is indexed by Science Citation Index (SciSearch), Scopus, International Pharmaceutical Abstract, Chemical Abstracts, Embase, Index Copernicus, EBSCO, African Index Medicus, JournalSeek, Journal Citation Reports/Science Edition, Directory of Open Access Journals (DOAJ), African Journal Online, Bioline International, Open-J-Gate and Pharmacy Abstracts

\section{INTRODUCTION}

Albendazole (ABZ), methyl [5-(propylthio)-I- Hbenzimidazol-2yl] carbamate, is considered as one of the most effective of the broad-spectrum anthelmintic agents [1]. The drug is very effective against systemic cestode infections especially in inoperable or disseminated cases of hydatidosis [2] and neurocysticercosis [3]. Low aqueous solubility may be the biggest problem hindering the systemic use of $A B Z$ [4]. Therefore, it is difficult to use $A B Z$ in the treatment of systemic helminthiasis [5], and so, it is important to enhance its poor aqueous solubility.

Aqueous solubility of the poorly soluble drugs could be enhanced by the application of auxiliary hydrophilic substances or by using new technological possibilities [6]. Solid dispersions of drugs in water-soluble carriers is of a great interest as a means of improving the dissolution 
rate, and hence possibly bioavailability, of a wide range of hydrophobic drugs [7].

Spray drying is a widely used technique to enhance drug solubility and dissolution rate [8]. Several drugs showed improved dissolution rates by spray drying of these drugs with hydrophilic polymers, as for example, indomethacin [9] and ketoprofen [10]. Kollicoat IR® $(\mathrm{KL})$ is a graft copolymer of poly (vinyl alcohol)-poly (ethylene glycol) (PVA-PEG) [11]. KL was used to enhance dissolution rates of poorly water-soluble drugs. Kollicoat IR-omeprazole microparticles with increasing its dissolution rate were prepared using spray- and freeze-drying techniques [12].

The aim of the present study is to enhance the dissolution rate of $A B Z$ for systemic absorption using co-spray-drying of $A B Z$ with Kollicoat and PVP in varying ratios.

\section{EXPERIMENTAL}

\section{Materials}

Albendazole (ABZ) was kindly donated from Saudi Pharmaceutical Industries (Riyadh, KSA). Kollicoat $I R \circledast(K L)$ was obtained from BASF (Ludwigshafen, Germany). Polyvinyl Pyrrolidone (PVP K30) was purchased from Flukachemica (Buch, Switzerland). All used materials were of analytical grades.

\section{Preparation of physical mixtures}

Physical mixtures (PMs) were prepared by gentle mixing the weighed amounts of $A B Z$ and each of the carriers (Kollicoat IR尺, PVP) in a mortar with a spatula. The ratios of the drug to the carrier used were 1:1, 1: 2and 1:4 by weight.

\section{Spray-dried binary systems using Kollicoat IR® and PVP}

Appropriate mass ratios $(1: 1,1: 2$, and $1: 4)$ of $A B Z$ with either $K L$ or PVP were prepared in water/ethanol (2: 1 mixture), where the drug was completely dissolved in ethanol, and $\mathrm{KL}$ or PVP was dissolved in distilled water. The aqueous solution was added gradually to the ethanolic drug solution with subsequent vigorous stirring for $1 \mathrm{~h}$ during spray-drying procedure to assure equilibrium. The resultant dispersion was spraydried in a Mini Spray-Dryer B-290 (BüchiLabortechnik AG, Flawil, Switzerland) with the following conditions: inlet temperature 140 ${ }^{\circ} \mathrm{C}$, outlet temperature $70-75^{\circ} \mathrm{C}$, the flow rate 5 $\mathrm{mL} / \mathrm{min}$, air flow rate $40-50 \mathrm{~m}^{3} / \mathrm{h}$, and atomizing air pressure $1.0-1.1$ bar. The batch size of the prepared ratios was about $5 \mathrm{~g}$ each.

\section{Drug loading}

The entrapment efficiency of $A B Z$ in the spraydrying particles was determined as the mass ratio of the entrapped drug to the theoretical amount of $A B Z$ used in the preparation. Spraydried particles equivalent to $5 \mathrm{mg}$ of drug were accurately weighed and dissolved in a suitable quantity of ethanol. The drug content was determined spectrophotometrically at $290 \mathrm{~nm}$.

\section{Morphological assessment}

The morphological characteristics of spray-dried particles were observed by scanning electron microscopy. The samples were sputter-coated with a thin gold palladium layer under an argon atmosphere using a sputter module in a highvacuum evaporator. The coated samples were then scanned and photomicrographs were taken with a JSM-1600 scanning electron microscope (Jeol, Tokyo, Japan).

\section{Differential scanning calorimetry (DSC)}

DSC studies of the drug and the spray-dried particles containing $A B Z$ in the matrices of $K L$ and PVP, as well as single components, were performed using a DSC-60 (Shimadzu, Kyoto, Japan). Samples $(3-5 \mathrm{mg})$ were placed in hermetically sealed aluminum pans. A scanning rate of $10^{\circ} \mathrm{C} / \mathrm{min}$ was used over the $25-200{ }^{\circ} \mathrm{C}$ temperature range. Indium was used as the temperature and enthalpy standard.

\section{Fourier transform infrared spectroscopy (FTIR)}

FTIR spectra of $A B Z$ and $A B Z$ binary systems with $\mathrm{KL}$ and $\mathrm{PVP}$ compared with the individual components were recorded using FTIR Perkin Elmer spectrophotometer (Spectrum BX). Samples were mixed with $\mathrm{KBr}$ and compressed into disks using hydraulic press before scanning from 4000 to $600 \mathrm{~cm}^{-1}$. The data were analyzed using Perkin Elmer software (Spectrum V5.3.1).

\section{In vitro dissolution studies}

The in vitro dissolution rate of $A B Z$ from its spray-dried systems in the matrices of $K L$ and PVP was investigated in a USP dissolution test apparatus (Caleva Ltd., Model 85T, Philips, UK). The dissolution profiles of $\mathrm{ABZ}$ from spray-dried systems were studied in $0.1 \mathrm{~N} \mathrm{HCl}(\mathrm{pH}=1.2)$. The drug-loaded particles equivalent to $20 \mathrm{mg}$ of $A B Z$ were placed in the dissolution vessel 
containing $500 \mathrm{ml}$ of the dissolution medium, which was rotated at $100 \mathrm{rpm}$ and temperature was kept constant at $37 \pm 0.5{ }^{\circ} \mathrm{C}$. At predetermined time intervals, the samples $(5 \mathrm{ml})$ were withdrawn, filtered and replaced immediately with fresh dissolution medium. The amount of drug dissolved was assayed spectrophotometrically at $290 \mathrm{~nm}$.

Dissolution efficiency after $15 \mathrm{~min}\left(\mathrm{DE} \%_{15}\right)$ was calculated from the area under the dissolution curve at time $t$ (measured using the trapezoidal rule) and expressed as percentage of the area of the rectangle described by $100 \%$ dissolution in the same time [13]. Also, the relative dissolution rate $\left(R D R_{15}\right)$ data of the different samples were calculated by determining the amount of $A B Z$ dissolved from a particular sample and normalizing for the amount of drug dissolved from pure drug sample over the same time interval (15 minutes).

\section{Statistical analysis}

The results were analyzed by using the software graph pad software (CA 92037 USA). Differences between formulations were considered to be significant at $p \leq 0.05$.

\section{RESULTS}

\section{Scanning electron microscopy (SEM)}

Scanning electron micrographs of $A B Z, K L$, spray-dried ABZ-KL and spray-dried ABZ-PVP systems in weight ratios of $1: 1$ and 1:4 compared to the corresponding physical mixtures are displayed in Fig. $1 A$ and $B A B Z$ exhibits irregularly shaped crystals, while $\mathrm{KL}$ particles appear highly spherical. In case of ABZ-physical mixtures with KL and PVP, drug crystals appear as lumpy aggregates surrounding the large polymer spheres.

Regarding the spray-dried drug-KL (1:2) mixture, the drug crystals began to appear as spherical and smaller and some of the polymer crystals appear large (Fig. 1B). On the other hand, the spray dried $A B Z-K L$ 1:4 revealed the presence of very small spheres of both drug and polymer that are seen homogeneous dispersed. In case of scanning electron images of spray-dried ABZPVP (1:1 and 1:4), the images showed that the drug crystals were homogeneously embedded as small-sized spheres- between the spherical polymer crystals (Fig. 1A).

\section{Thermal analysis}

The DSC thermogram of pure ABZ (Fig. 2 A) shows an endothermic peak at $218{ }^{\circ} \mathrm{C}$ with a shoulder at $198{ }^{\circ} \mathrm{C}$ due to drug melting [15], while $\mathrm{KL}$ exhibits a broad endotherm at 213.14 ${ }^{\circ} \mathrm{C}$ with a thaw point of $207.5^{\circ} \mathrm{C}$. The drug's melting shoulder was shallow and broad and its melting endotherm disappeared in case of its-KL physical mixtures in weight ratios of $1: 1$ and $1: 4$. On the other hand, ABZ endotherm has been no longer detected in case of the drug-KL spraydried mixtures at the studied ratios (1:1 and 1:4) and the drug melting shoulder was seen broad in case of 1:1 ratio.

The DSC thermograms of ABZ-PVP binary systems are illustrated in Fig. 2B. It was clear that PVP showed a shallow, broad endothermic peak around $81{ }^{\circ} \mathrm{C}$, which represents the vaporization of moisture from the PVP sample. DSC scan of ABZ-PVP 1:1 physical mixture showed that the endothermic peak of the drug existed at the same position compared to the untreated drug but it lost its distinct sharpness, while in the case of ABZ-PVP 1:1 physical mixture, the drug melting endotherm has been no longer seen.

\section{Fourier transform infrared spectroscopy (FTIR)}

FTIR spectra of $A B Z$ binary systems with $K L$ and PVP compared with the individual components are displayed in Figs $3 \mathrm{~A}$ and $\mathrm{B}$. The spectrum of the $A B Z$ shows an absorption peak of stretching vibration, characteristic of the $\mathrm{NH}$ bond as a band of medium intensity at $3323 \mathrm{~cm}^{-1}$ and a bending vibration at $1525 \mathrm{~cm}^{-1}$. In addition, stretching bands and deformation characteristics of aliphatic carbons were seen at $2958 \mathrm{~cm}^{-1}$ and $1450 \mathrm{~cm}^{-1}$, respectively. Moreover, a stretching band of the aromatic ring double bonds appeared at $1590 \mathrm{~cm}^{-1}$, in addition to a strong peak of stretching vibration corresponding to the $\mathrm{C}=\mathrm{O}$ group at $1634 \mathrm{~cm}^{-1}$. The FTIR spectrum of $\mathrm{KL}$ showed a characteristic broad band at $3421 \mathrm{~cm}^{-}$ 1 , which is assigned for $\mathrm{OH}$ stretching and a stretching vibration band of $\mathrm{C}=\mathrm{O}$ at $1637 \mathrm{~cm}^{-1}$ as well as a $\mathrm{C}-\mathrm{H}$ stretching vibration peak at 2915 $\mathrm{cm}^{-1}$. PVP showed a characteristic stretching $\mathrm{C}=\mathrm{O}$ band at $1673 \mathrm{~cm}^{-1}$.

\section{In vitro dissolution profiles of $A B Z$ from $K L$ and PVP binary systems}

Fig. 4 shows the in vitro dissolution profiles of $A B Z$ from its-KL spray-dried systems using

Trop J Pharm Res, December 2014; 13(12): 1965 
A
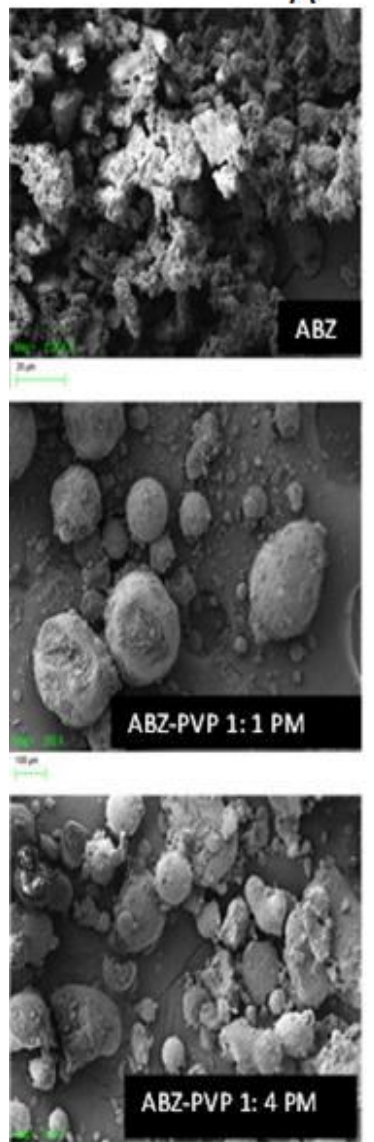
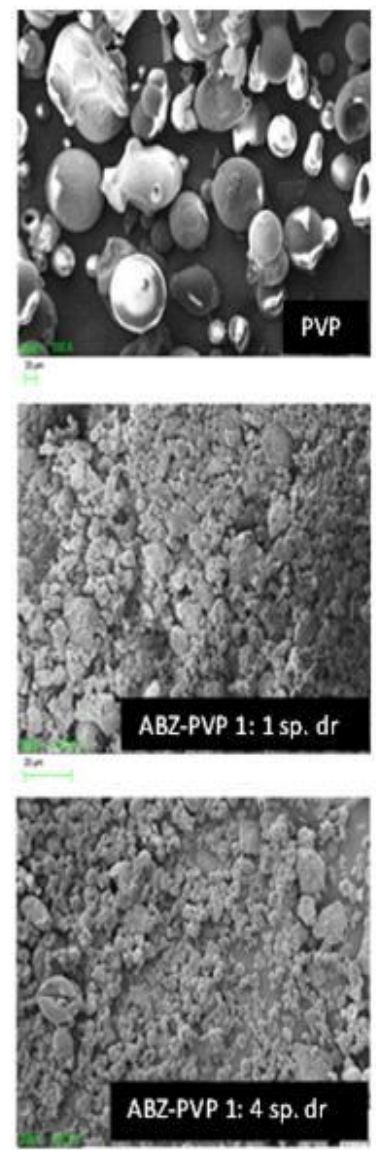

B
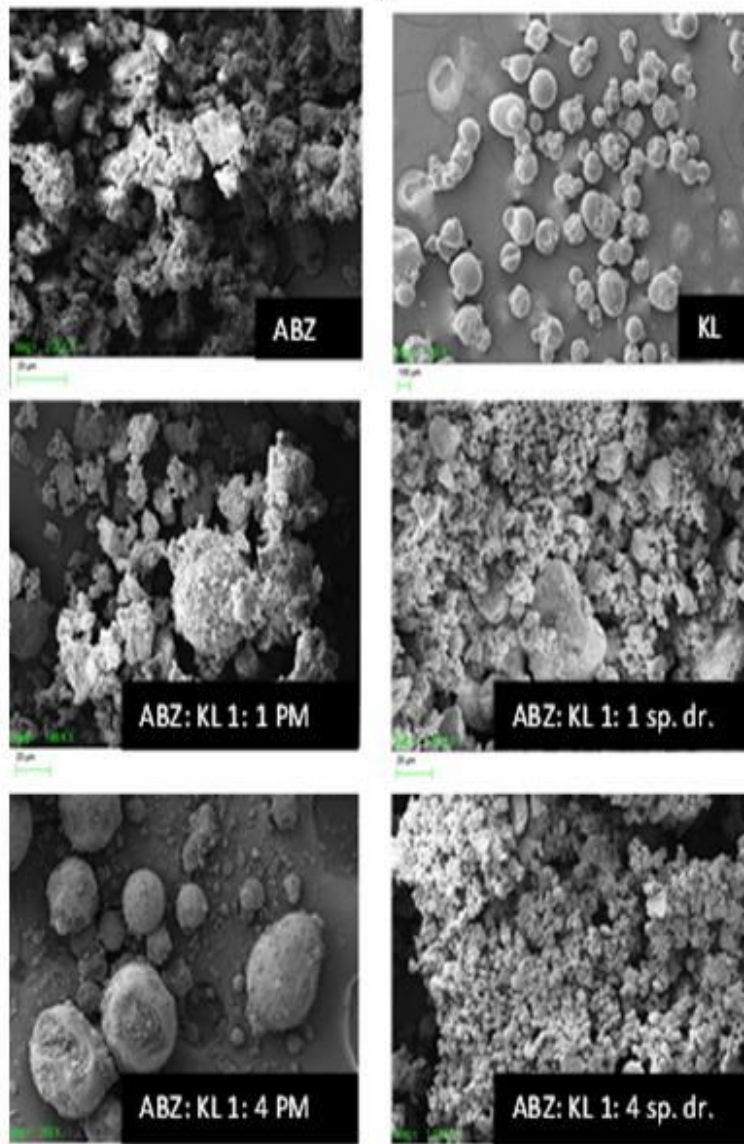

Fig 1: $\mathbf{A}=\mathrm{SE}$ micrographs of $\mathrm{ABZ}-\mathrm{KL}$ spray dried and physical mixtures in drug: polymer ratios $1: 1$ and $1: 4$ compared to the individual components; $\mathbf{B}=\mathrm{SE}$ micrographs of ABZ-PVP spray dried and physical mixtures in drug: polymer ratios 1: 1 and 1: 4 compared to the individual components

A

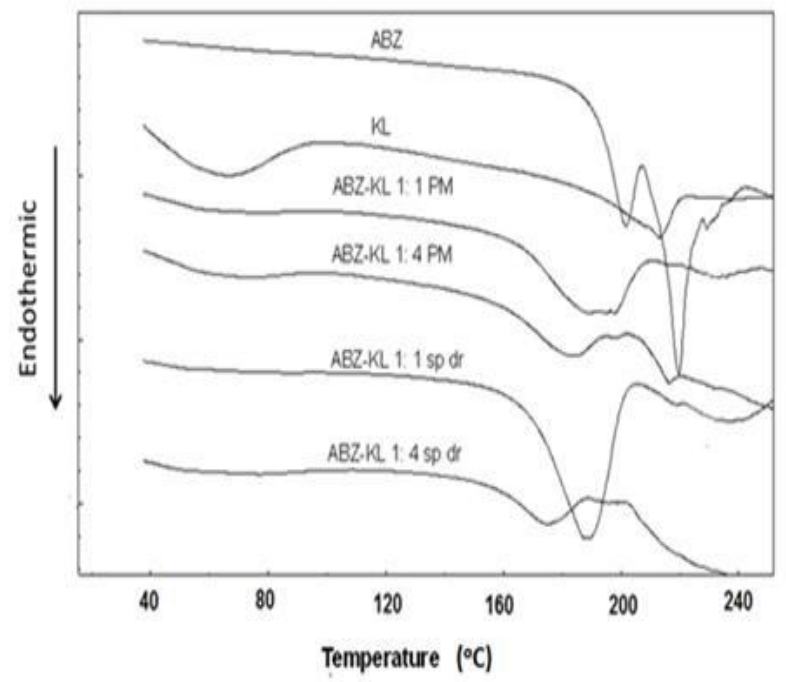

B

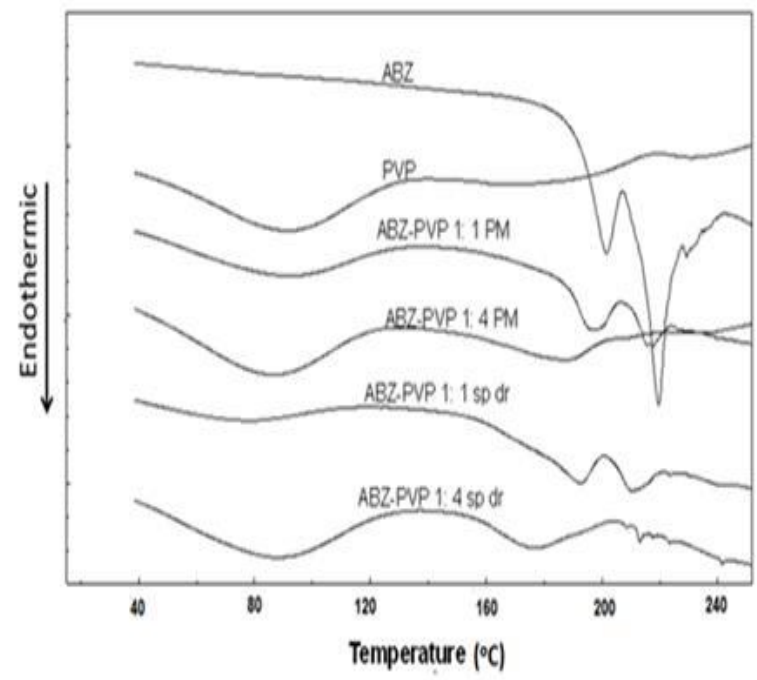

Fig 2: $A=D S C$ thermograms of $A B Z-K L$ spray dried and physical mixtures in drug: polymer ratios $1: 1$ and $1: 4$ compared to the individual components, and B = DSC thermograms of ABZ-PVP spray dried and physical mixtures in drug: polymer ratios 1: 1 and 1: 4 compared to the individual components 

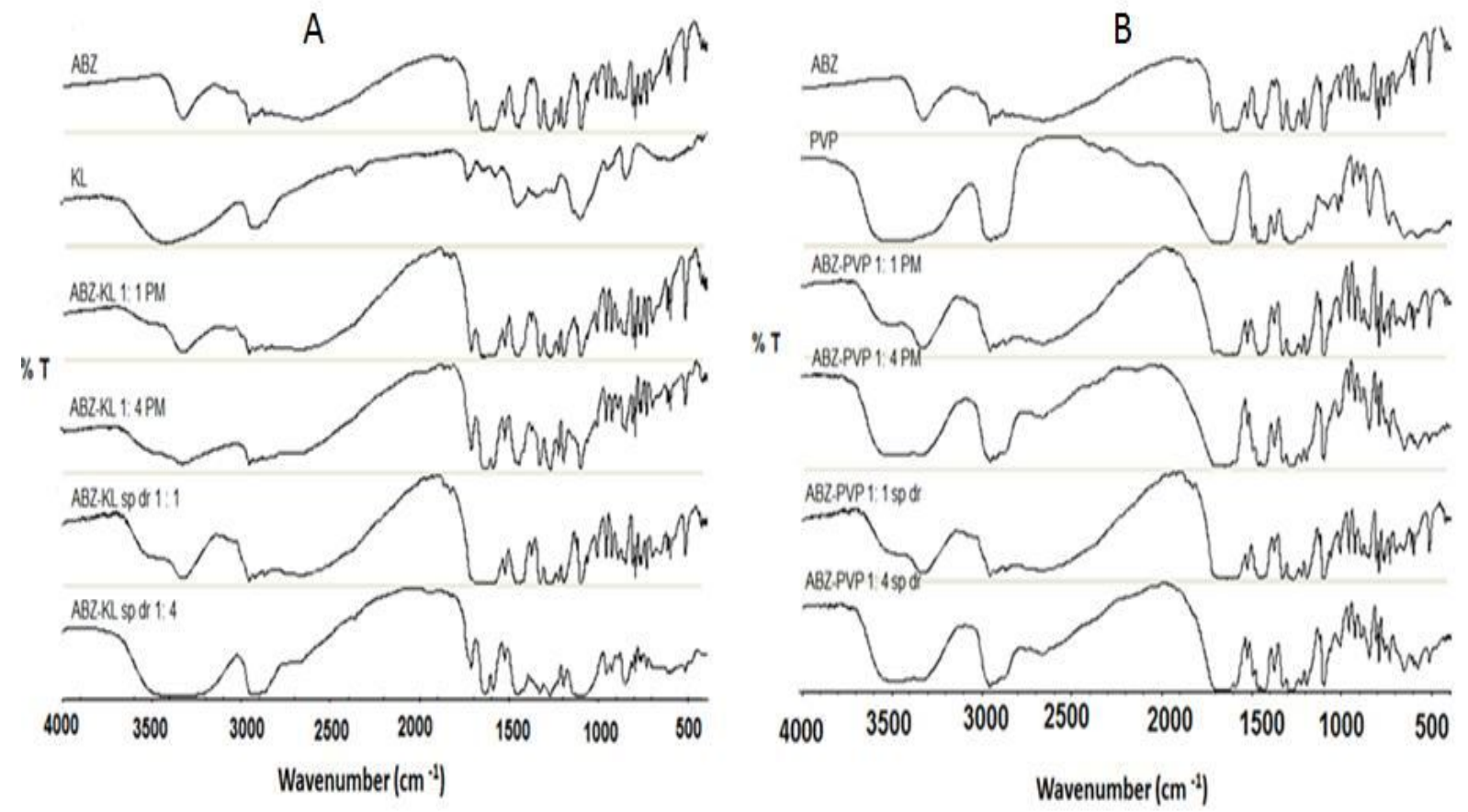

Fig 3: $A=$ FTIR spectra of $A B Z-K L$ spray dried and physical mixtures in drug: polymer ratios 1: 1 and $1: 4$ compared to the individual components, and B = FTIR spectra of ABZ-PVP spray dried and physical mixtures in drug: polymer ratios 1: 1 and 1: 4 compared to the individual components

different drug: polymer ratios. $A B Z$ alone exhibited slow dissolution rate, in which only 8.8 $\%$ were dissolved within $15 \mathrm{~min}$., and about $25 \%$ were dissolved after $60 \mathrm{~min}$. Spray-drying of the drug in $\mathrm{KL}$ matrix caused a noticeable enhancement of its dissolution rate under the influence of $A B Z$ : $K I$ ratio. Upon using 1:1 ratio, the drug dissolution rate was slightly improved with $\mathrm{D} \%{ }_{15}$ and $\mathrm{RDR}_{15}$ values of 18.43 and 4.31 , respectively (Table 1). These values are not significantly different from the values recorded in case of 1:1 and 1:4 physical mixtures from which the drug exhibited $D_{15}$ values of 20.67 and 25.84 , respectively and $R^{2} R_{15}$ values of 4.94 and 5.61, respectively. However, in case of increasing the polymer weight ratio (1:2 and 1:4 spray-dried systems), the drug's dissolution was remarkably enhanced. For example, $100 \%$ and $96 \%$ of $A B Z$ was dissolved within $30 \mathrm{~min}$ from spray-dried systems using drug: $K L$ ratios of $1: 2$ and $1: 4$, respectively, and, in turn, the drug $\mathrm{RDR}_{15}$ was increased nine folds in both ratios (Table 1). It is clearly evident that the drug dissolution rate from its-KL spray dried particles (1:2 and 1:4 ratios) was statistically different from that observed in case of using $A B Z-K L$ ratio of $1: 1(p<0.05)$.
The dissolution patterns of ABZ-PVP spray-dried systems were displayed in Fig. 5. Comparatively, the dissolution enhancement of ABZ from itsPVP spray-dried systems was higher than that observed in case of its-KL systems. In addition, the extent of the dissolution enhancement was not significantly governed by the polymer weight ratio (only the release from ratio $1: 1$ is significantly different from that obtained from the ratio $1: 2$ at the first $30 \mathrm{~min}, p<0.05$ ). The drug was completely dissolved within $15 \mathrm{~min}$ from itsPVP spray-dried systems in case of drug: polymer ratios of $1: 1$ and $1: 2$, while about $97 \%$ of the drug amount was dissolved after the same time from ABZ-PVP 1:4 ratio. Furthermore, the values of $D \%_{15}$ and $R D R_{15}$ recorded in case of 1:1, 1:2 and 1:4 ABZ: PVP ratios were very close (74.07, 76.29 and $72.66 \mathrm{D} \%_{15}$ values were observed for 1:1, 1:2 and 1:4 ABZ-PVP spraydried systems, respectively (Table 1). Moreover, a very slight increase in the drug dissolution rate was recorded in case of physical mixtures with PVP using 1:1 and 1:4 ABZ: PVP ratios. However, the release of $A B Z$ from its-PVP spraydried systems is significantly different from that recorded in case of the corresponding physical mixtures $(p<0.05)$. 
Table 1: Dissolution efficiency (DE\% 15$)$ and relative dissolution rates after 15 minutes (RDR $\left.{ }_{15}\right)$ of $A B Z$ from its spray-fried $\mathrm{KL}$ and PVP systems and some physical mixtures

\begin{tabular}{lcc}
\hline System & $\mathbf{D E}_{\mathbf{1 5}}$ & $\mathbf{R D R}_{\mathbf{1 5}}$ \\
\hline ABZ & 4.44 & - \\
ABZ-KL spray dried (1:1) & 18.43 & 4.31 \\
ABZ-KL spray dried (1:2) & 45.24 & 9.17 \\
ABZ-KL spray dried (1:4) & 52.99 & 9.52 \\
ABZ-KL Physical Mixture (1:1) & 4.94 \\
ABZ-KL Physical Mixture (1:4) & 20.67 & 5.61 \\
ABZ-PVP spray dried (1:1) & 25.84 & 12.05 \\
ABZ-PVP spray dried (1:2) & 74.07 & 12.53 \\
ABZ-PVP spray dried (1:4) & 76.29 & 11.37 \\
ABZ-PVP Physical Mixture (1:1) & 72.66 & 3.08 \\
ABZ-PVP Physical Mixture (1:4) & 14.34 & 3.84 \\
\hline
\end{tabular}

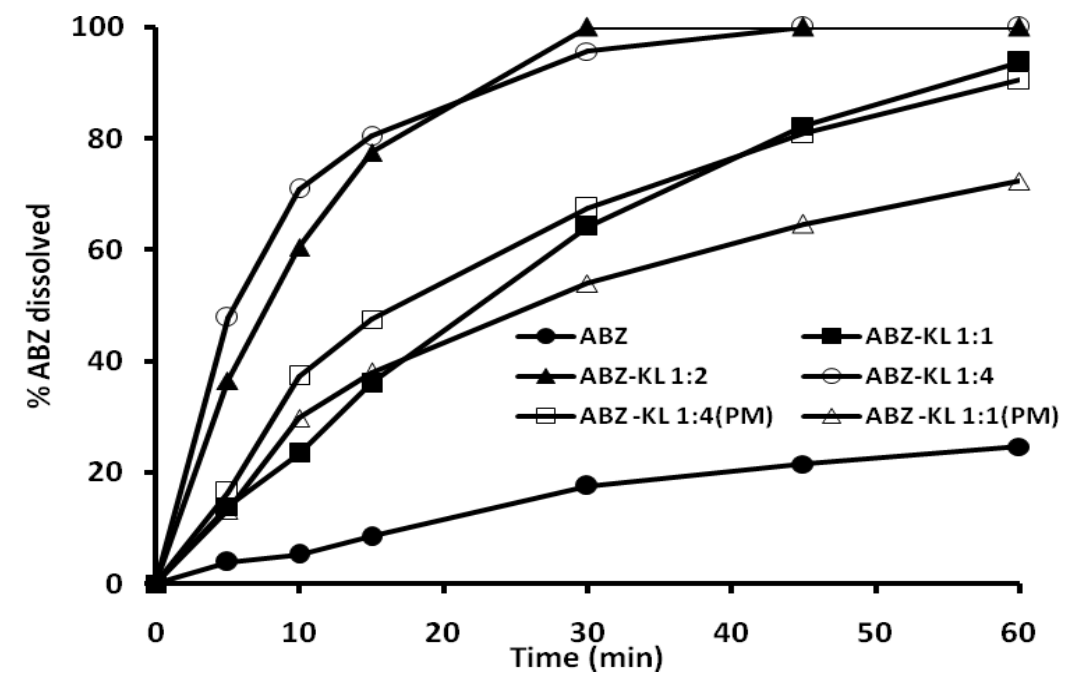

Fig 4: Dissolution profiles of $A B Z-K L$ spray-dried systems compared to the corresponding physical mixtures (PM)

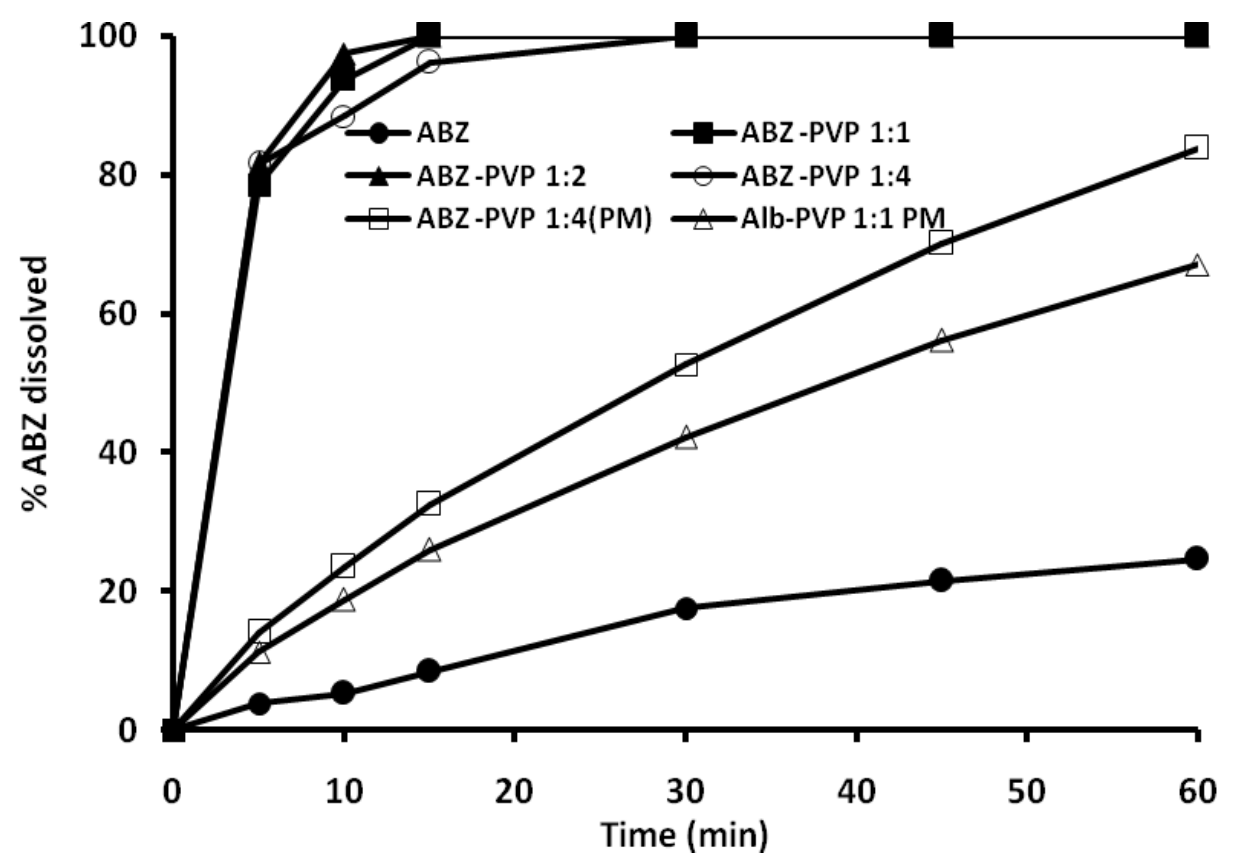

Fig 5: Dissolution profiles of ABZ-PVP spray-dried systems, and physical mixtures (PM) 


\section{DISCUSSION}

The DSC thermograms of spray-dried ABZ with either KL or PVP at high polymer ratios showed disappearance of the melting endotherm of the drug, indicating homogenous drug dispersion in the polymeric matrices [14]. In addition, some drug-polymer physical mixtures showed the disappearance of the melting endotherm, and this could be attributed to the solubility of $A B Z$ in the molten polymers. Same findings were observed by Ibrahim and Al-Anazi [15], who found that the disappearance of $A B Z$ melting endotherm in case of the drug-physical mixtures with lactose and microcrystalline cellulose and they attributed this finding to the solubility of $A B Z$ in the molten polymers.

The FTIR spectra of ABZ-KL and ABZ-PVP systems of both physical mixtures and spraydried mixtures did not show any change of either the drug or the polymer characteristic bands in terms of the position or intensity, which proves the compatibility of the used carriers with $A B Z$.

\section{In vitro dissolution profiles of $A B Z$ from $K L$} and PVP binary systems

In comparing the dissolution rate of $A B Z$ alone with its spray-dried systems in KL matrix, $A B Z$ alone exhibited slow dissolution rate, while a noticeable enhancement of its dissolution rate was observed under the influence of $A B Z: K L$ ratio. It was found that by increasing the polymer weight ratio, the drug dissolution was remarkably enhanced. The enhancement of dissolution of $A B Z$ was found to be higher in its-PVP spray dried systems compared to $\mathrm{KL}$ systems. Moreover, a very slightly increased in the drug's dissolution rate was recorded in case of physical mixtures with PVP using 1:1 and 1:4 ABZ:PVP ratios.

The enhancement of $A B Z$ dissolution rate might be due to improving the wettability of the drug particles and by significantly reducing the drug particle size during the spray-drying with hydrophilic matrices. Fouad et al [16] showed that the increased dissolution rate of celecoxib in spray-dried mixtures of $\mathrm{KL} \quad \mathrm{IR}$ and other excipients was due to improving drug wettability. Also, the inherently higher dissolution rate of the soluble polymer introduces the less-soluble drug as finely divided particles into the dissolution medium [17]. Furthermore, Janssens et al [18] explained that a significant decrease of the drug particle size, possibly to the molecular level, in combination with an additional effect of the codissolving hydrophilic carrier on the drugs solubility, might decrease the time required for complete dissolution.

It is clearly from the drug dissolution profiles that the drug spray-dried in PVP matrices (in different weight ratios) showed an enhanced drug dissolution rates that were higher than those observed in case of ABZ-KL systems. This finding is in agreement with that of Van den Mooter et al [19], who found that PVP was effective in preventing drug crystallization when the drug was formulated as solid dispersions in PVP matrices. They concluded therefore that the physical mechanism of the protective effect of PVP in the case of amorphous ketoconazole is not the consequence of drug-polymer interactions, but mainly due to the polymer antiplasticizing effect, thereby increasing the viscosity of the binary system and decreasing the diffusion of drug molecules necessary to form a lattice. Moreover, that PVP enhanced ABZ dissolution rate even in low polymer weight ratio $(1: 1)$ rather than KL. It could be seen from the SEM images of ABZ-PVP spray-dried system using drug to polymer ratio 1:2 showed homogeneous distribution of both the drug and polymer matrices, while in case of ABZ-KL 1:2 spray-died system, the drug and polymer particles were not seen homogeneous.

The data obtained from DSC, FTIR and SEM studies demonstrated that the spray-drying of $A B Z$ in the matrices of both $K L$ and PVP enhanced its dissolution rate and this enhancement could not be attributed to drugcarries interaction. The hydrophilic polymers improved the drug particles' wettability, by reducing the drug particle size during spraydrying. The action of embedding the finely divided drug particles in the matrices of the soluble component should also be considered. This is in accordance with El Badry et al [20], who showed that a total omeprazole amorphosization was induced by spray-drying and freeze-dried processes for both Kollicoat $I R \circledast$ and HP- $\beta-C D$ confirmed that the drug is no longer present in crystalline form and it was changed to amorphous state.

\section{CONCLUSION}

The dissolution rate of $A B Z$ could be enhanced by spray-drying technique using hydrophilic polymers such as $\mathrm{KL}$ and PVP. The mechanism of drug dissolution enhancement can be attributed to the hydrophilic nature of the polymers used, production of small-sized spherical particles by spray-drying and change of 
the drug crystals embedded in the polymeric matrices to amorphous form.

\section{ACKNOWLEDGEMENT}

The authors extend their appreciation to the Deanship of Scientific Research at King Saud University for funding the work through Research Group Project no. RGP - VPP - 139.

\section{REFERENCES}

1. Cook GC. Use of benzimidazole chemotherapy in human helminthiases: indications and efficacy. Parasitol Today 1990; 6: 133-136.

2. Wen H, New RRC, Craig PS. Diagnosis and treatment of human hydatidosis. Br J Clin Pharm 1993; 35: 56557.

3. Del-Brutto $\mathrm{OH}$, Sotelo J, Roman GC. Therapy for neurocysticercosis: a reappraisal. Clin Infect Dis 1993; 17: 730-735.

4. Torrado S, Torrado S, Torrado JJ, Cadórniga $R$. Preparation, dissolution and characterization of Albendazole solid dispersions. Int J Pharm 1996, 140: 247-250.

5. Yasawy MI, Al-Karawi MA, Mohamed AR. Combination of praziquantel and albendazole in the treatment of hydatic diseases. Trop Med Parasitol 1993; 44: 192194.

6. Jachowicz R, Nürnberg E, Pieszczek B, Kluczykowska B, Maciejewska $A$. Solid dispersion of ketoprofen in pellets. Int J Pharm 2000; 206: 13-21.

7. Craig DQM. The mechanisms of drug release from solid dispersions in water-soluble polymers. Int $J$ Pharm 2002; 231: 131-144.

8. Alanazi FK, El-Badry M, Ahmed MO, Alsarra IA. Improvement of albendazole dissolution by preparing microparticles using spray-drying technique. Sci Pharm 2007; 75: 63-79.

9. Alanazi FK, El-Badry M, Alsarra IA. Spray-dried HPMC microparticles of indomethacin: impact of drugpolymer ratio and viscosity of the polymeric solution on dissolution. Saudi Pharm J 2006; 14: 100-107.
10. Moretti MD, Gavini E, Juliano C, Pirisino G, Giunchedi $P$. Spray-dried microspheres containing ketoprofen formulated into capsule and tablets. J Microencapsul 2001; 18: 111-121.

11. DeMerlis CC, Schoneker DR. Review of the oral toxicity of polyvinyl alcohol (PVA). Food Chem Toxicol 2003; 41: 319-326.

12. EL-Badry M, Alanazi FK, Mahrous GM, Alsarra IA. Effects of Kollicoat IRß and hydroxypropyl- $\beta$ cyclodextrin on the dissolution rate of omeprazole from its microparticles and enteric-coated capsules. Pharm Dev Technol 2010; 15: 500-510.

13. Khan KA. The concept of dissolution efficiency. J Pharm Pharmacol 1975; 27: 48-49.

14. Alanazi FK, El-Badry M, Ahmed MO, Alsarra IA. Improvement of Albendazole Dissolution by Preparing Microparticles Using Spray-Drying Technique. Sci Pharm 2007; 75: 63-79.

15. Ibrahim MA, Al-Anazi FK. Enhancement of the Dissolution of Albendazole from Pellets Using MTR Technique. Saudi Pharm J 2013; 21: 215-223.

16. Fouad EA, EL-Badry M, Mahrous GM, Alanazi FK, Neau $\mathrm{SH}$, Alsarra IA. The use of spray-drying to enhance celecoxib solubility. Drug Dev Ind Pharm 2011; 37 : 1463-1472.

17. Passerini N, Perissutti $B$, Moneghini $M$, Voinovich $D$, Albertini B, Cavallari $C$ et al. Characterization of carbamazepine-gelucire 50/13 microparticles prepared by a spray-congealing process using ultrasounds. J Pharm Sci 2002; 91: 699-707.

18. Janssens $S$, Anné $M$, Rombaut $P$, Van den Mooter $G$. Spray drying from complex solvent systems broadens the applicability of Kollicoat IR as a carrier in the formulation of solid dispersions. Eur J Pharm Sci 2009; 37: 241-248.

19. Van den Mooter G, Wuyts M, Blaton B, Busson R, Grobet $P$, Augustijns $P$, Kinget $R$. Physical stabilisation of amorphous ketoconazole in solid dispersions with solid dispersions with PVP K25. Eur J Pharm Sci 2001; 12:261-269.

20. EL-Badry M, Alanazi $F K$, Mahrous GM, Alsarra IA. Effects of Kollicoat IR® and hydroxypropyl- $\beta$ cyclodextrin on the dissolution rate of omeprazole from itsmicroparticles and enteric-coated capsules, Pharm Dev Technol 2010; 15(5):500-510. 